\title{
Response-outcome versus outcome-response associations in instrumental learning
}

\author{
ROBERT A. RESCORLA \\ University of Pennsylvania, Philadelphia, Pennsylvania
}

\begin{abstract}
In three experiments with rats as subjects, instrumental training procedures were used to study the format of encoding of the reinforcing outcome $(0)$. At issue is the relative contribution of response-outcome (R-O) associations between responses and their earned outcomes and of $O-R$ associations between anticipated outcomes and responses reinforced in their presence. In Experiments 1 and $2, \mathrm{R}$ earned one $\mathrm{O}$ during a stimulus that controlled the anticipation of another $O$. Devaluation and transfer tests suggested that the earned $O$ was more critical than the anticipated $\mathrm{O}$ in controlling behavior. In Experiment 3, a differential-outcomes procedure was used, with consistent $\mathrm{R}-\mathrm{O}$ relations arranged in groups that differed in the consistency of $\mathrm{O}-\mathrm{R}$ relations. Subsequent devaluation of $O$ produced similar selective depression of $R$, regardless of the $O-R$ relations. These results suggest that an $R-O$ association can contribute to instrumental performance more than does an $\mathrm{O}-\mathrm{R}$ association.
\end{abstract}

There is now strong evidence that instrumental training routinely results in the formation of an association between the response and its outcome. Outcomes do not simply act to strengthen associations between responses and other stimuli; they are themselves encoded in the course of instrumental training.

In earlier research from this laboratory, two techniques have been used to investigate such associations between responses and outcomes. One technique involves the revaluation of an outcome after it has been used to train a response (see, e.g., Colwill \& Rescorla, 1985; Rescorla, 1990). Such outcome revaluation produces changes in performance that are specific to the responses which that outcome has previously trained. Since the revalued outcome is not presented during response testing, this implies that prior training has resulted in an association between it and the specific response. The second technique involves the transfer of a discriminative stimulus trained with one response to the control of another response (see, e.g., Colwill \& Rescorla, 1988). That transfer is successful to the degree that the same outcome is used with the stimulus and with the transfer response. An instrumental response shows little enhancement in the presence of a discriminative stimulus trained with another outcome. That outcome specificity implies that the response has become associated with the particular outcome used to train it.

Colwill and Rescorla $(1985,1988)$ have argued that these results are naturally interpreted in terms of an $\mathrm{R}-\mathrm{O}$ association between a response and the outcome that follows it. However, one could also view such results in

This research was supported by National Science Foundation Grant BNS-88-03514. Correspondence concerning this article should be addressed to Robert A. Rescorla, Department of Psychology, University of Pennsylvania, 3815 Walnut Street, Philadelphia, PA 19104. terms of an $\mathrm{O}-\mathrm{R}$ association, such as that emphasized by some forms of the historically popular two-process theory (see, e.g., Trapold \& Overmier, 1972). According to such a view, stimuli present during instrumental training develop an association with the outcome and hence control the anticipation of $O$. Subsequently, responding is reinforced in the presence of that anticipation of $O$, resulting in an $\mathrm{O}-\mathrm{R}$ association.

Such an O-R theory can account for the essential features of both the revaluation and the transfer findings that have otherwise been interpreted in terms of an $\mathrm{R}-\mathrm{O}$ association. Devaluation of $\mathrm{O}$ may hinder performance because it adversely affects processing of one of the primary stimuli controlling responding. Similarly, transfer may occur because a discriminative stimulus trained with an $\mathrm{O}$ evokes the anticipation of that $\mathrm{O}$ which in turn evokes the transfer response.

Moreover, the two-process theory has generated an impressive number of experiments showing the importance of differential outcomes in controlling discrimination performance. Beginning with the initial work of Trapold (1970), a great many experiments have shown superior discrimination performance in a task in which each of two stimuli signals the reinforcement of a particular response if those responses are reinforced by different outcomes (see, e.g., Peterson, Linwick, \& Overmier, 1987; Peterson, Wheeler, \& Armstrong, 1978). According to the twoprocess account, this differential outcomes effect results because each $S$ becomes associated with a unique $O$, which in turn becomes associated with a particular $R$. The presence of a common $\mathrm{O}$ in the $\mathrm{S}-\mathrm{O}$ and $\mathrm{O}-\mathrm{R}$ associations helps mediate superior performance, especially under circumstances in which the discrimination is made more difficult by imposing a temporal gap between $\mathrm{S}$ and $\mathrm{R}$.

Of course, such an effect can also be understood in terms of O's being common to an S-O and an $\mathrm{R}-\mathrm{O}$ associ- 
ation. Indeed, the differential outcomes effect can be viewed as the converse of the transfer of stimulus control across responses shown by Colwill and Rescorla (1988). The use of different outcomes for responses reduces the transfer to the alternative response of the stimulus being trained with a particular response. Since that transfer is inappropriate, its reduction facilitates the selective performance required during the stimulus. Nevertheless, the broad range of experiments demonstrating this effect originated in the context of the two-process theory, and mediation by an O-R association has remained the dominant interpretation.

There have been some attempts to evaluate the relative contributions of an R-O and an O-R association to instrumental performance. For instance, Rescorla and Colwill (1989) conducted a series of experiments in which responses were reinforced by one outcome, $\mathrm{Ol}$, but that reinforcement occurred at a time when external stimuli led the animal to anticipate another outcome, O2. Consequently, an association of the form $\mathrm{O}-\mathrm{R}$ would involve $\mathrm{O} 2$, whereas one of the form $\mathrm{R}-\mathrm{O}$ would involve $\mathrm{O} 1$. They then carried out devaluation and transfer experiments to identify whether $\mathrm{O} 1$ or $\mathrm{O} 2$ was encoded in the association with $\mathrm{R}$. Their findings indicated that $\mathrm{R}$ was well associated with the consequent $\mathrm{Ol}$ and associated less well, if at all, with the anticipated $\mathrm{O} 2$. Consequently, their results suggested an encoding in the form of $\mathrm{R}-\mathrm{O}$.

The experiments reported here were intended to continue the investigation of these alternative ways of encoding the outcome. They were designed to increase the opportunities for the formation of O-R associations. In Experiments 1 and 2, the strategy of Rescorla and Colwill (1989) was followed: a response was reinforced with one outcome at a time when another was anticipated. Experiments 1 and 2 differed from the earlier experiments in that the opportunity for $\mathrm{O}-\mathrm{R}$ learning was maximized by extensive reinforcement of $\mathbf{R}$ with $\mathrm{O}$ 1. In Experiment 1 , this control was assessed with a devaluation technique, whereas in Experiment 2, this was done with a transfer technique. Experiment 3 had a somewhat different strategy. A version of a differential outcomes experiment was arranged, in which two groups both had consistent $\mathbf{R}-\mathrm{O}$ relations but differed in the consistency of their $\mathrm{O}-\mathrm{R}$ relations. At a point at which a differential outcomes effect was being observed, and hence when those $\mathrm{O}-\mathrm{R}$ associations might have been functioning differentially in two groups, a devaluation operation was conducted. If devaluation effects are mediated by $\mathrm{O}-\mathrm{R}$ associations, they should be more substantial in the consistent group; if they are mediated by $\mathrm{R}-\mathrm{O}$ associations, they should be similar in the two groups.

All of the experiments employed rat subjects in standard instrumental training procedures using auditory and visual signals that various responses would be reinforced by appetitive outcomes such as pellets and liquid sucrose.

\section{EXPERIMENT 1}

Experiments 1 and 2 were extensions of experiments previously reported by Rescorla and Colwill (1989). They sought to separate $\mathrm{R}-\mathrm{O}$ and $\mathrm{O}-\mathrm{R}$ alternatives by deliberately arranging for one $\mathrm{O}$ to be a frequently anticipated event at the time when a response was reinforced by another $O$. One can then ask about the relative importance of the various Os when devaluation and transfer procedures are used. When Rescorla and Colwill used such a strategy, they found evidence that $\mathrm{R}-\mathrm{O}$ associations dominated the form of encoding.

The present experiments constituted an attempt to extend their findings in two ways. First, in an attempt to maintain the original $\mathrm{S}-\mathrm{O}$ association, Rescorla and Colwill (1989) used a procedure in which a response was followed by $\mathrm{O} 1$ only a few times in the presence of a stimulus that had previously signaled $\mathrm{O} 2$. As a result of such minimal training, the stimulus never actually gained control over the target response. Consequently, the opportunity for the formation of $\mathrm{O} 2-\mathrm{R}$ associations might have been relatively limited. In the present experiments, much more extensive training was used, to allow repeated reinforcement of a response in the presence of an outcome anticipation. Second, in the Rescorla and Colwill experiments, the stimulus signaling the outcome had been trained as a discriminative stimulus for another response. It seems possible that such a stimulus is not the optimal one for producing the anticipation of a particular outcome. Consequently, in the present studies, the anticipation was instead provided by a well-trained Pavlovian signal of the outcome.

Figure 1 schematizes the design used in Experiment 1. All animals were originally trained to make two responses, leverpress and chainpull (R1 and R2), to obtain an irrele-

\begin{tabular}{l|c|c|c|c} 
Pretrain & Pavlovion & Discrimination & Devaluation & Test \\
\hline R1-03 & S1-01 & S1: R1-02; 01 & 01-LiCl & S1S2: R1 V R2 \\
R2-03 & S2-02 & S2: R2-01; 02 & &
\end{tabular}

Figure 1. Design of Experiment 1. Two stimuli, S1 and S2, were given Pavlovian conditioning with different outcomes, $O 1$ and $O 2$. With this treatment continued, $S 1$ and $S 2$ were used as discriminative stimuli signaling that responding would yield other outcomes. After one outcome was paired with $\mathrm{LiCl}$, a choice was given between the responses in the presence of the S1S2 compound. 
vant common outcome (cherry pellet). This was done to encourage a high rate of responding in subsequent phases without providing any experience with the outcomes of interest. Then each animal was given simple Pavlovian conditioning in which one outcome occurred during a light and the other during a noise, both independently of responding. Next, each animal was rewarded for responding on the lever and the chain in the presence of the light and noise. However, the outcome that each response earned was the opposite of that used in Pavlovian training of the signal. Moreover, during that training, the original Pavlovian outcome continued to occur at a higher rate during the stimulus than did the earned outcome. In order to evaluate whether the outcome expected during the stimulus or that earned by the response would become better associated with the response, one outcome was devalued in each animal, and a test was given for responding during a compound of the light and noise.

\section{Method}

\section{Subjects and Apparatus}

The subjects were 16 male Sprague-Dawley rats about 90 days old. They were housed in individual cages and maintained on a food deprivation regime that kept them at $80 \%$ of their ad-lib body weights. They had free access to water in their home cages.

The apparatus consisted of eight operant chambers measuring 22.9 $\times 20.3 \times 20.3 \mathrm{~cm}$, identical to those used in previous reports (e.g., Colwill \& Rescorla, 1985). The two end walls of each chamber were made of aluminum; the sidewalls and ceiling were made of clear Plexiglas. Each chamber had a recessed food magazine in the center of one end wall. A small metal cup measuring $1.25 \mathrm{~cm}$ in diameter and $1.5 \mathrm{~cm}$ deep was sunk in the floor of each food magazine. Four different manipulanda could be made available in each chamber. To the left of the magazine was a lever and to the right was a chain suspended from a microswitch mounted on the lid of the chamber. Located directly above the food magazine was a $2-\mathrm{cm}$ opening behind which was an aluminum plate that activated an attached microswitch when displaced by a nosepoke. Located under the grid floor just to the right of the magazine aperture was a handlepull manipulandum. This consisted of a small flat rod with the end bent back to form a handle; an upward pull on the handle operated a microswitch. Access to these manipulanda could be blocked by covering the lever with a metal shield, retracting the chain through a hole in the ceiling, covering the nosepoke opening with a jeweled lens, and removal of the handlepull. The floor of the chamber was composed of $0.48-\mathrm{cm}$ stainless steel rods, spaced $1.9 \mathrm{~cm}$ apart. Outside the chamber, near the rear wall, a 6-W bulb was mounted at the level of the grid floor. This light could be illuminated at a rate of $1 / \mathrm{sec}$ to provide a flashing light (F) stimulus.

Each chamber was enclosed in a sound- and light-resistant shell. Mounted on the inside wall of this shell were speakers that permitted the presentation of a white noise $(\mathrm{N})$ and an $1800-\mathrm{Hz}$ tone $(\mathrm{T})$, each measuring approximately $76 \mathrm{~dB}$ re $20 \mu \mathrm{N} / \mathrm{m}^{2}$ against a background level of $62 \mathrm{~dB}$. Also mounted on that wall was a 6-W bulb that could be illuminated to provide a light $(\mathrm{L})$ stimulus during the otherwise dark session. The outside ceiling of the shell supported a solenoid-operated gravity feed valve that was connected via plastic tubing to the cup in the food magazine. This system permitted the presentation of $.3 \mathrm{ml}$ of an $8 \%$ sucrose solution. Also attached to that food magazine was a dispenser containing 45-mg pellets (either Formula A or cherry pellets from P. J. Noyes Co.).

Experimental events were controlled and recorded automatically by relays and microprocessors located in an adjoining room.

\section{Procedure}

Initial training. All of the animals received magazine training with three outcomes. On the 1st day, all the animals received 10 deliveries of the standard pellets and then 10 of the sucrose, delivered at a mean rate of $1 / \mathrm{min}$. On the 2 nd day, they received 10 deliveries of the cherry pellets. No response manipulanda were available during these sessions. On the next 2 days, the animals were trained to leverpress and chainpull. In each session, one manipulandum was made available, and the animals were allowed to earn 25 cherry pellets on a continuous reinforcement schedule. On the next day, all the animals received two 20-min sessions, spaced about an hour apart, during each of which responding was reinforced on a variable interval (VI) 60 -sec schedule. The lever was present in the first session, and the chain was present in the second. In each session, the outcome earned consisted of cherry pellets.

Pavlovian conditioning. On each of the next 14 days, all of the animals received Pavlovian conditioning of both the $\mathrm{L}$ and $\mathrm{N}$ in the absence of any manipulandum. Each session contained sixteen $30-\mathrm{sec}$ presentations of each stimulus, during which a variable time (VT) 30-sec reinforcement schedule was in effect. For half of the animals, sucrose was delivered during $\mathrm{N}$ and standard pellets during $\mathrm{L}$; for the remaining half of the animals, the reinforcement contingencies were reversed. The intertrial interval (ITI) was variable around a mean of $120 \mathrm{sec}$. On the next day, all animals received another day on which both responses were retrained on the VI schedule, using the cherry pellet.

Discrimination training. On each of the next 12 days, all of the animals received discrimination training in which the lever and chain allowed them to earn outcomes in the presence of $\mathrm{L}$ and $\mathrm{N}$, in the manner schematized in Figure 1. Each day contained two sessions, one with the lever present and one with the chain present. During each session, one of the stimuli was presented 16 times for $30 \mathrm{sec}$. The outcome used to train that stimulus in the Pavlovian phase continued to occur on a VT $30-\mathrm{sec}$ schedule in its presence. In addition, the other outcome could be earned by the response on a VI 30 -sec schedule. Response identities were counterbalanced across stimuli and outcomes. The mean ITIs were 45, 60, 90, and $120 \mathrm{sec}$ for two, two, four, and four sessions, respectively.

Outcome devaluation. Next, the animals received five 2-day cycles intended to devalue one of the outcomes. Throughout this period, all manipulanda were removed from the chambers and one outcome was paired with a $0.5 \%$ body weight $.6 \mathrm{M} \mathrm{LiCl}$ i.p. injection. On the 1st day of each cycle, one outcome was programmed for delivery 20 times at a rate of $1 / \mathrm{min}$. At the end of the session, the animal was removed from the chamber, given the $\mathrm{LiCl}$ injection, returned to its home cage, and fed $2 \mathrm{~h}$ later. On the 2 nd day of each cycle, the procedure was the same with the other outcome, but no injection was given. In the fourth and fifth cycles, the doors on the isolation chambers were left open to permit observation of the animals. On those days, an animal was removed and given its injection as soon as five reinforcer deliveries were left unconsumed. The choice of which outcome was paired with $\mathrm{LiCl}$ was orthogonal to the previous treatments.

Test. On the next day, the animals received a test session during which both manipulanda were available. During that session, they received eight 30 -sec presentations of the NL compound, spaced with a mean ITI of $120 \mathrm{sec}$. The intention of testing with the LN compound was to ensure reasonably high levels of overall performance while guaranteeing the presence of anticipations of both $\mathrm{Os}$ at the time of testing.

\section{Results and Discussion}

Initial response training and Pavlovian conditioning were uneventful. Discriminative performance developed steadily during the discrimination phase. By the final day 


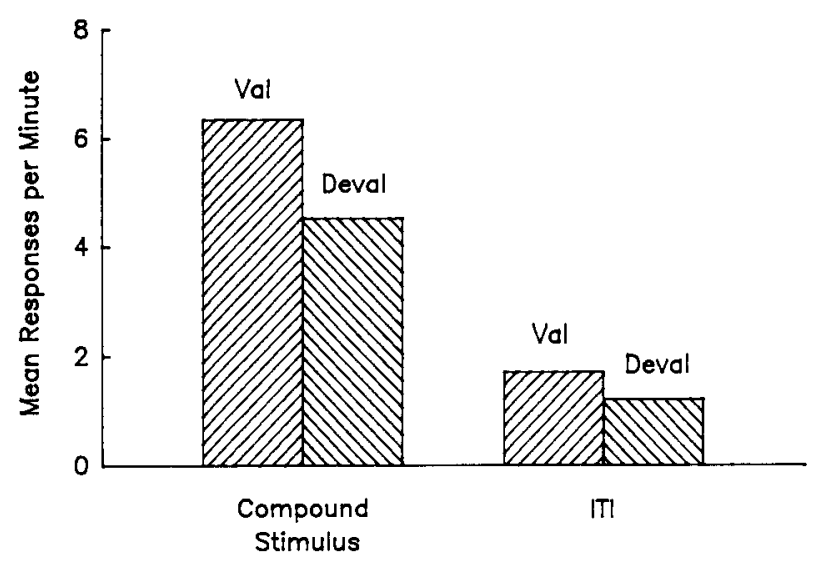

Figure 2. Mean responses per minute during the test in Experiment 1 . Performance is shown for the response whose earned outcome had been devalued or remained valuable, both in the presence of the compound stimulus and in the intertrial interval.

of training, the mean numbers of responses per minute were 8.0 and 2.4 during the stimuli and the ITI, respectively. A discrimination ratio of the form $x /(x+y)$, where $x$ and $y$ are response rates in the stimulus and ITI, respectively, showed a mean of .74 . That ratio was reliably different $[T(16)=2, p<.01]$ from one of .5 . There were only minor differences as a function of stimulus, response, and outcome identity. However, despite the equivalent parameters of the VI and VT schedules, the freely delivered outcome occurred with substantially greater frequency throughout this phase. For instance, on the final day of discrimination training, the mean number of earned reinforcers was 8.5 over 16 trials, whereas the mean number of freely delivered reinforcers was at the programmed rate of 16 over the 16 trials. This discrepancy resulted because during signaled periods of VI availability, the animals routinely failed to earn all of the programmed reinforcers. The consequence was that each stimulus continued throughout discrimination training to best signal the outcome with which it was paired during Pavlovian conditioning.

Devaluation of the outcomes was rapid and nearly complete. On the final day of this phase, the animals consumed all of the nonpoisoned outcomes but none of the poisoned outcomes.

The results of most interest, from the test session with the compound stimulus, are shown in Figure 2. The figure shows the mean rate of responding during the stimulus and in the ITI, for the response whose earned outcome had been devalued or not. Notice that because of the experimental design, devaluing the earned outcome of a response meant leaving valued the freely delivered outcome. Hence the animal was faced with a choice between two responses, one with a consequent outcome devalued and anticipated outcome left valuable, and one with a consequent outcome left valuable and anticipated outcome devalued. It is clear that during the compound stimulus, the response whose consequence had been devalued was made with a lower frequency than that of the response whose consequence had retained its value $[T(16)=27$, $p<.05]$. A similar pattern is observed in the absence of any discriminative stimulus, but the overall level of responding is too low to permit a serious evaluation.

These results suggest that the response was more sensitive to the devaluation of the outcome that it actually earned, compared with that which the stimulus signaled at the time that the response was reinforced. That result occurred under circumstances in which training was carried on sufficiently long to allow the stimulus to gain control over the response. Therefore, it provided ample opportunity for the anticipated outcome to develop an association with the response. Consequently, these results suggest that the findings reported by Rescorla and Colwill (1989) are not confined to the early portion of learning. Under circumstances intended to maximize the potential contribution of $\mathrm{O}-\mathrm{R}$ associations, devaluation procedures still suggest a more substantial contribution of the $\mathrm{R}-\mathrm{O}$ association.

\section{EXPERIMENT 2}

This experiment was a companion to Experiment 1 in which the encoding of the outcome was assessed by a transfer, rather than a devaluation, procedure. As in Experiment 1 , the animals were trained to earn one outcome in the presence of a Pavlovian signal of another outcome. However, unlike in Experiment 1, the determination of which outcome was encoded in conjunction with the response was made by presenting discriminative stimuli that had signaled the outcomes for another pair of responses.

The design of Experiment 2 is presented schematically in Figure 3. In the manner of Experiment 1, two different responses ( $R 1$ and $\mathrm{R} 2$ ) earned different outcomes $(\mathrm{O} 1$ and $\mathrm{O} 2$ ) in the presence of two visual Pavlovian stimuli

\begin{tabular}{l|c|c|c|c} 
Pretrain & Discrimination & Pavlovian & Discrimination & Test \\
\hline $\begin{array}{l}\text { R1-03 } \\
\text { R2-03 }\end{array}$ & S3:R3-01 & S1-01 & S1: R1-02; 01 & S3: R1 V R2 \\
R3-03 & S4:R4-02 & S2-02 & S2: R2-01; 02 & S4: R1 V R2 \\
R4-03 & S4
\end{tabular}

Figure 3. Design of Experiment 2. Two stimuli, S1 and S2, were given Pavlovian conditioning with different outcomes, $\mathrm{O1}$ and $\mathrm{O2}$. With this treatment continued, S1 and S2 were used as discriminative stimuli signaling that $R 1$ and $R 2$ would yield the alternative outcomes. Then a choice was given between $R 1$ and $R 2$ in the presence of two other stimuli, S3 and S4, which had previously served as signals of the outcomes for different responses. 
(S1 and S2), each of which otherwise signaled the alternative outcome. Prior to that training, two auditory stimuli (S3 and S4) were trained with other responses (R3 and R4) as instrumental signals of $\mathrm{O} 1$ and $\mathrm{O} 2$. These auditory stimuli were then transferred to R1 and R2. The question was, which response would be augmented by a discriminative stimulus that signaled $\mathrm{O} 1-$ a response that had earned $\mathrm{O} 1$ in the presence of the anticipation of $\mathrm{O} 2$, or a response that had earned $\mathrm{O} 2$ in the presence of the anticipation of 01 ? The former result would suggest that an $\mathrm{R}-\mathrm{O}$ association mediated transfer, whereas the latter would suggest that an $\mathrm{O}-\mathrm{R}$ association did so.

\section{Method}

\section{Subjects and Apparatus}

The subjects were of the same type and were maintained in the same manner as in Experiment 1. The apparatus was that of Experiment 1 , modified so as also to permit .3-ml deliveries of a $5 \%$ Polycose solution. Polycose is an outcome that rats find highly attractive and distinguish readily from sucrose solution and pellets.

\section{Procedure}

Initial training. On each of the first 3 days, the animals received magazine training with all manipulanda removed from the chambers. They received 20 deliveries each of pellets, sucrose, and Polycose, at a rate of $1 / \mathrm{min}$, in the first, second, and third sessions, respectively.

On the next 2 days, the animals received response training with the lever and then the chain in the same manner as in Experiment 1 , except that a $5 \%$ Polycose solution was always the reinforcer. On each of the next 5 days, the animals received two 20-min VI training sessions per day, one with the lever and one with the chain. In all sessions, the outcome was Polycose, delivered on a VI 60 $\mathrm{sec}$ schedule. This training procedure was then repeated for 2 continuous reinforcement and 3 VI training days, with nosepoke and handlepull as the manipulanda.

Discrimination training. On each of the next 10 days, the animals received discrimination training designed to establish $\mathrm{T}$ and $\mathbf{N}$ as discriminative signals of sucrose and pellets. Each day contained two sessions spaced about $1 \mathrm{~h}$ apart, one with nosepoke and one with handlepull available. In each session, sixteen 30-sec presentations of either $\mathbf{T}$ or $\mathbf{N}$ were given, and either sucrose or pellets were available for responding on a VI 30 -sec schedule. The roles of the responses and outcomes were counterbalanced across animals, but for any particular animal, $\mathrm{T}$ and $\mathrm{N}$ always signaled a particular outcome for a particular response. The ITIs were 45 , 60 , and $90 \mathrm{sec}$ for the first 3 days of training; thereafter, the ITI was $120 \mathrm{sec}$.

Pavlovian conditioning. On each of the next 10 days, the animals received training designed to establish each of the visual stimuli as a Pavlovian signal for a particular outcome. Each session contained sixteen 30 -sec presentations each of steady light (L) and flashing light $(F)$, each signaling the occurrence of a particular reinforcer on a VT 30-sec schedule. In a manner orthogonal to the previous treatments, for half of the animals $\mathrm{L}$ signaled pellets and F signaled sucrose; for the other half of the animals, the contingencies were reversed. No manipulanda were available during these sessions.

Training of target response. On the next day, all animals received retraining of the lever and chain, with Polycose delivered on a VI schedule in the manner of their initial training. Then the animals received 10 days of discrimination training with the lever and chain. Two sessions were scheduled on each day; the sessions were separated by about an hour, one with sixteen 30-sec presentations of $\mathrm{L}$ and one with sixteen 30 -sec presentations of $F$. During each session, the stimulus continued to signal Pavlovian deliveries of its outcome on the VT 30-sec schedule. But in addition, in each session, either the lever or the chain was available to the animal, and responding during the stimuli earned the other outcome on a VI 30-sec schedule. The ITIs were 45, 60, and $90 \mathrm{sec}$ for the first 3 days; thereafter, the ITI was $120 \mathrm{sec}$. The choice of responses present during the visual stimuli was counterbalanced with regard to prior treatments.

Test. On the next 3 days, the animals received retraining of the auditory discriminations with nosepoke and handlepull, in the manner of their prior treatment. Then the animals received three test sessions, during each of which they were given a choice between the lever and the chain. Each session was $\mathbf{8}$ min long and contained 430 -sec deliveries each of $T$ and $N$, spaced $30 \mathrm{sec}$ apart. No visual stimuli were presented during these sessions.

\section{Results and Discussion}

Initial response training proceeded smoothly, as did the auditory discrimination in which nosepoke and handlepull earned either sucrose or pellets during $\mathbf{N}$ and $\mathrm{T}$. By the final day of discrimination training, the mean responses per minute were 21.7 and 5.4 during the stimuli and ITI, respectively. Discrimination training with the lever and chain during $L$ and $F$ developed well. By the final day of training, the mean responses per minute were 8.9 and 1.1 during the stimuli and the ITI, respectively. The terminal discrimination ratio was .78. Throughout this phase of training, the outcome delivered in a Pavlovian fashion routinely occurred more frequently than did that earned by responding. On the final day of discrimination training, the mean number of outcomes earned per 16 trials was 11.2, whereas the mean number delivered on the VT schedule was 16 in 16 trials.

The results of most interest, from the transfer tests in which the auditory stimuli were presented while the animal chose between the lever and the chain, are shown in Figure 4. That figure shows the results combined across all stimuli and test sessions, but separated according to whether the stimulus had signaled the same outcome as, or a different outcome from, that which the response had

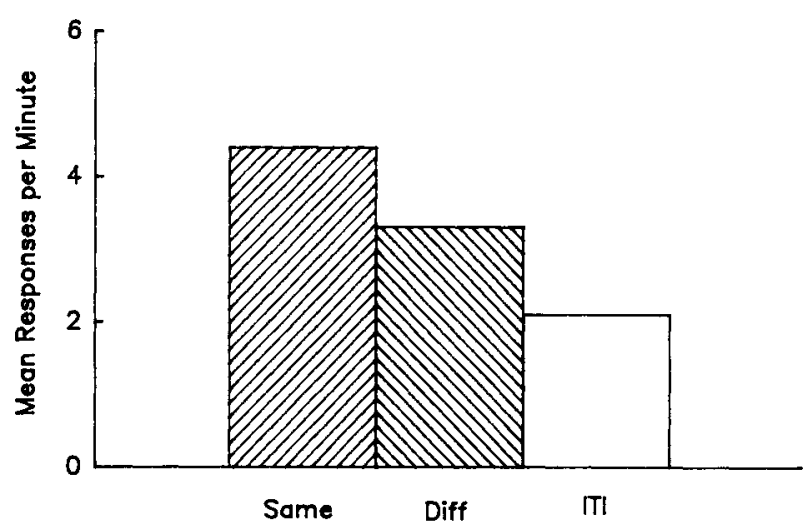

Figure 4. Mean responses per minute during the test sessions of Experiment 2. Responding is shown in the intertrial interval and in the presence of stimuli that signaled the same or a different outcome from that which the response had earned. 
earned. Notice that, because of the experimental design, a stimulus designated as different with respect to the consequent outcome also signaled the outcome that had been delivered on the Pavlovian schedule. It is clear from that figure that responding was elevated more substantially by the stimulus that signaled the same (i.e., the earned) outcome compared with the stimulus that signaled the different (i.e., freely delivered) outcome $[T(15)=17.5$, $p<.02$ ]. However, the stimulus signaling the different outcome also elevated responding relative to that in the ITI $[T(16)=1, p<.01]$.

These results from a transfer test agree with those from the devaluation procedure used in Experiment 1. They suggest that the earned outcome contributes more to transfer than does the outcome anticipated when that response is reinforced. That is, they favor the importance of an $\mathrm{R}-\mathrm{O}$ compared with an O-R format of outcome encoding.

\section{EXPERIMENT 3}

In Experiments 1 and 2, R-O and O-R associations were compared with the transfer and devaluation procedures that have commonly been interpreted as demonstrating $\mathrm{R}-\mathrm{O}$ associations. In those experiments, the attempt was made to maximize the opportunities for $\mathrm{O}-\mathrm{R}$ associations during instrumental learning by guaranteeing the presence of anticipations of $O$ at the time $R$ was reinforced. Experiment 3 instead used a differential outcomes discrimination procedure whose results are routinely interpreted in terms of $\mathrm{O}-\mathrm{R}$ associations. Within a twoprocess theory, such a differential outcomes effect is evidence for the effective presence of $\mathrm{O}-\mathrm{R}$ associations. One may then ask whether under those circumstances such associations would control the results of a devaluation procedure.

In a typical differential outcomes experiment, two groups of animals learn a discrimination in which S1 signals that $R 1$ will be reinforced and $S 2$ signals that $R 2$ will be reinforced. However, for a consistent group, $\mathrm{S} 1$ and $\mathrm{S} 2$ also signal unique outcomes, $\mathrm{O} 1$ and $\mathrm{O} 2$, whereas for an inconsistent group, $\mathrm{O} 1$ and $\mathrm{O} 2$ each occur for responding in S1 and S2, in an unpredictable fashion. The typically observed result is that the consistent group acquires the discrimination more rapidly.

According to the two-process (O-R) account, the slower acquisition on the part of the inconsistent group occurs because there is no consistent association between either the stimuli and outcomes or the outcomes and the responses. The inconsistent training results in both $\mathrm{S} 1$ and $\mathrm{S} 2$ becoming associated with both $\mathrm{O} 1$ and $\mathrm{O} 2$. As a result, both R1 and R2 are reinforced in the presence of anticipations of both $\mathrm{O} 1$ and $\mathrm{O} 2$. By contrast, in the consistent group, $\mathrm{S} 1$ becomes associated only with $\mathrm{O} 1$ and $S 2$ only with $\mathrm{O} 2$. Then $\mathrm{R} 1$ and $\mathrm{R} 2$ are only reinforced in the presence of anticipations of $\mathrm{O} 1$ and $\mathrm{O} 2$, respectively. The S1 only evokes the anticipation of $\mathrm{O} 1$, and $\mathrm{Ol}$ is only associated with $\mathrm{R} 1$; the result is that there is little tendency for $\mathrm{S} 1$ to evoke $\mathrm{R} 2$ inappropriately through the mediation of the anticipated outcomes.

Although it is less common, an interpretation of these results can also be given in terms of an $\mathrm{R}-\mathrm{O}$ association. On that account, the important inconsistency is that between the response and the following outcome rather than that between the response and the outcome anticipated when it is reinforced. That is, performance can be seen as partially mediated by the $\mathrm{S}-\mathrm{O}$ association activating a representation of $O$, which in turn activates a response by means of the "backward" action of an R-O association. In other regards, the argument remains the same as for the two-process account.

One way to separate these two accounts is to arrange for the same discriminative stimuli to control more than one pair of responses. Consider the design used in Experiment 3, illustrated in Figure 5. In this design, all animals are taught two discriminations, one between $\mathrm{R} 1$ and $\mathrm{R} 2$ and one between R3 and R4, but both controlled by

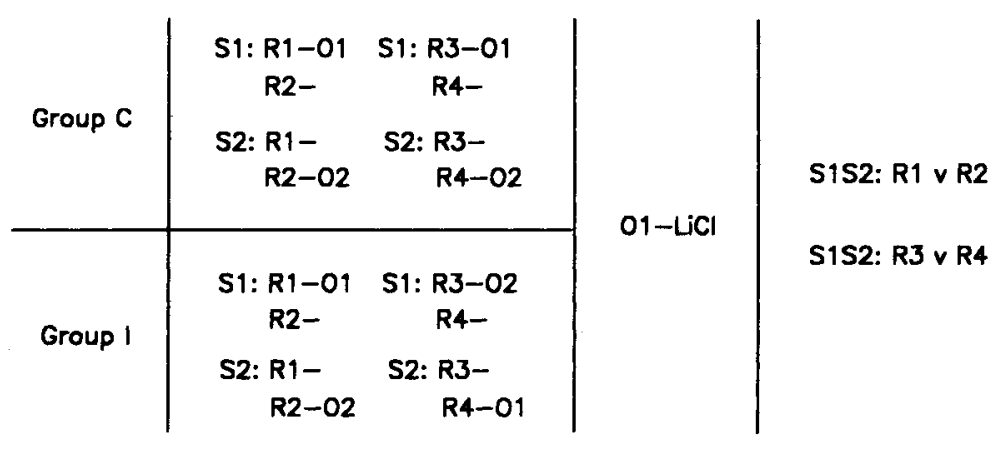

Figure 5. Differential outcomes design used in Experiment 3. All animals learned to discriminate between two pairs of responses-R1 and R2, and $R 3$ and $R 4-$ in the presence of two stimuli, S1 and S2. For Group C (consistent), each S signaled the same outcome ( $O$ ) across both discriminations; for Group I (inconsistent), each $S$ signaled a different $O$ in the two discriminations. For both groups, each $R$ always earned the same $\mathrm{O}$. One $\mathrm{O}$ was then paired with $\mathrm{LiCl}$, and both response pairs were tested in the S1S2 compound. 
S1 and S2. For Group C (consistent), differential outcomes are consistently associated with $S 1$ and $S 2$, as well as with the particular responses. For Group I (inconsistent), however, $\mathrm{S} 1$ and $\mathrm{S} 2$ signal, respectively, $\mathrm{O} 1$ and $\mathrm{O} 2$ for the R1/R2 discrimination, but they signal, respectively, $\mathrm{O} 2$ and $\mathrm{O} 1$ for the $\mathrm{R} 3 / \mathrm{R} 4$ discrimination.

Both of the accounts under consideration anticipate that the discriminations will be learned more rapidly in Group C. However, they differ in the basis of that prediction. For the two-process theory, there are two sources of difficulty for Group I. Neither $S$ is consistently associated with a particular outcome, and, as a consequence, neither $\mathrm{R}$ is consistently reinforced in the presence of the anticipation of a particular outcome. That is, according to a two-process description, each $S$ is associated with both Os and both Os are associated with each R. However, an account that emphasizes the importance of $\mathrm{R}-\mathrm{O}$ associations identifies only one source of difficulty for Group I. According to that view, each $S$ is indeed associated with both Os, but each response is consistently followed by a particular outcome, even in Group I.

Consequently, the two views see these manipulations as having quite different implications for the degree to which a particular $R$ is associated with a particular $O$. According to the two-process account, Group $\mathrm{C}$, but not Group I, has a unique association between $R$ and $O$. According to the alternative account, both groups have equivalent associations between $R$ and $O$. One could then hope to separate the two alternatives by asking about the selectivity of changes in the value of the outcomes for the two groups. According to an approach that describes the outcome as encoded in terms of an $\mathrm{O}-\mathrm{R}$ association, Group $C$ should show a highly selective effect of devaluing the outcome used with each response, but Group I should show a less selective effect. However, according to an approach that describes the outcome as encoded in terms of an $\mathrm{R}-\mathrm{O}$ association, the two groups should show equivalent selectivity.

\section{Method \\ Subjects and Apparatus}

The subjects were 16 rats of the same type, maintained in the same manner as in Experiment 1. The apparatus was that of Experiment 1.

\section{Procedure}

Initial training. On the 1st day, the animals received a single 20-min magazine training session, during which first 10 pellets and then 10 sucrose deliveries occurred, spaced a mean of 1 min apart. Over the next 4 days, all animals were trained to leverpress, chainpull, nosepoke, and handlepull. Each training session made one manipulandum available, and responding earned 25 deliveries of the appropriate outcome on a continuous reinforcement schedule. Half of the animals received one session in which leverpressing led to pellets and one in which chainpulling led to sucrose; for the other half of the animals, the contingencies were reversed. Similarly, an orthogonally chosen half of the animals received one session in which nosepoking led to pellets and one in which handlepulling led to sucrose, whereas the other half of the animals had the contingencies reversed. Throughout this initial training, individual shaping was used if necessary for a particular $\mathrm{R}-\mathrm{O}$ combination. On each of the next 2 days, all animals received two 20-min sessions, spaced about an hour apart, during each of which responding was reinforced on a VI 60 -sec schedule. On the 1st day, the lever was present in the first session and the chain was present in the second. On the 2nd day, the nosepoke and handlepull were present in the first and second sessions, respectively. The outcome earned on the VI schedule was the same for each response as that used in continuous reinforcement training.

Discrimination training. On each of the next 2 days, the animals received two discrimination sessions, spaced about an hour apart. Each session contained sixteen $30-\mathrm{sec}$ presentations of either $\mathrm{N}$ or L, with a mean ITI of $45 \mathrm{sec}$. During each session, only one of the manipulanda to be reinforced during a stimulus was actually present, and responding earned the same outcome as had been used during VI training. On the lst day, the manipulanda present were the lever and chain; on the 2 nd day, they were handlepull and nosepoke.

On each of the next 5 days, the animals received discrimination training sessions during which two manipulanda were present concurrently (either lever and chain or handlepull and nosepoke), and both $\mathrm{L}$ and $\mathrm{N}$ were presented. On each day, the animals received two sessions, each containing 16 presentations each of $L$ and of $\mathrm{N}$. During each stimulus, one response was rewarded according to a VI 30-sec schedule, whereas the other was not rewarded. The ITIs were $45,60,90,120$, and $120 \mathrm{sec}$, on successive days.

As indicated in Figure 5, for Group $\mathrm{C}$ the two stimuli each signaled the availability of a particular outcome, whichever response pair was present. The actual identities of the stimuli, responses, and outcomes were counterbalanced across animals. However, for Group I, each stimulus signaled the availability of one outcome with one response pair and the alternative outcome with the other response pair. Again the identities of the stimuli, responses, and outcomes were counterbalanced. But for both groups a particular response always earned a particular outcome whenever it was reinforced. During acquisition, the question of interest was whether the inconsistency of $\mathrm{S}-\mathrm{O}$ relations would yield retarded learning, despite the consistency of $\mathrm{R}-\mathrm{O}$ relations in both groups.

Outcome devaluation. Next, the animals received outcome devaluation in the same manner as in Experiment 1. The choice of which outcome was paired with $\mathrm{LiCl}$ was arranged to be orthogonal to the previous treatments.

Test. On each of the next 2 days, the animals received a single test session during which eight 30-sec trials with the LN compound were given with a mean ITI of $120 \mathrm{sec}$. During the first test session, the lever and chain were present; during the second test session, the nosepoke and handlepull were present. The intention of testing with the LN compound was to ensure reasonable levels of responding while matching the effects of the $\mathrm{LiCl}$ treatment on the stimuli present during testing in the two groups. It also guarantees the anticipation of both outcomes during the stimuli and consequently should maximally expose the use of either $\mathrm{R}-\mathrm{O}$ or $\mathrm{O}-\mathrm{R}$ associations. The question of interest was whether the effect of devaluation on the responses would be more substantial in Group $C$, which had consistent O-R relations, compared with Group I, which had inconsistent $\mathrm{O}-\mathrm{R}$ relations.

\section{Results}

Initial response training proceeded smoothly. Figure 6 shows the course of discrimination training. The results are plotted in terms of discrimination ratios, of the form $x /(x+y)$, where $x$ is the response rate during the stimulus and $y$ is that in the ITI. The results have been combined across the counterbalancing but are shown separately for the response reinforced $(+)$ and nonreinforced $(-)$ in Groups C and I. Over the course of training, performance improved in both groups, with the response reinforced 
in each stimulus showing an elevation and that nonreinforced in each stimulus showing a decrease, compared with responding in the ITI. Both changes were reliable for both groups of animals [Wilcoxon $T s(8)=0, p s<.01$ ]. Of more interest was that the discriminative performance was superior in Group C. This was especially clear in its more rapid loss of responding to the nonreinforced response. On the final day of discrimination training, Group C showed reliably greater suppression of that response [Mann-Whitney $U(8,8)=10, p<.05$ ]. The rate of responding on the reinforced alternative in each stimulus was not reliably different between the two groups.

In order to allow comparisons with previous experiments, Figure 6 shows responding to the various stimuli using a discrimination ratio relative to the ITI, in the manner of Experiments 1 and 2. However, in this experiment, several responses were concurrently present and the discrimination is solved in terms of choosing the appropriate response during each stimulus. Consequently, the data may also be described in terms of the relative rates of responding on the correct and incorrect manipulanda during a stimulus. Such a description yields similar conclusions. For instance, on the last day of discrimination training, the percentage of trial responses that were on the correct manipulanda were 90 and 82 , for Group $\mathrm{C}$ and Group I, respectively $[U(8,8)=12, p<.05]$. That is, these data reveal a conventional differential outcomes effect.

The devaluation of the outcomes proceeded smoothly. By the final day of devaluation, the animals consumed a mean of less than one of the devalued outcomes, but all consumed all of the nondevalued outcomes.

The results of most interest, from the postdevaluation test with the compound stimuli, are shown in Figure 7. Because there were no differences as a function of response identity, the results have been combined across

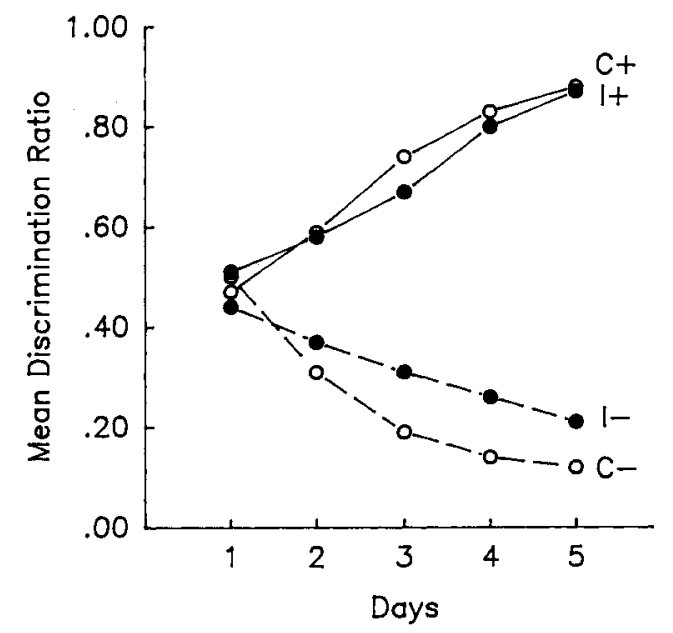

Figure 6. Discrimination training phase of Experiment 3. Discrimination ratio comparing rate during the stimulus with that in the intertrial interval is shown for Groups $C$ and $I$, separately for the reinforced $(+)$ and nonreinforced $(-)$ responses.

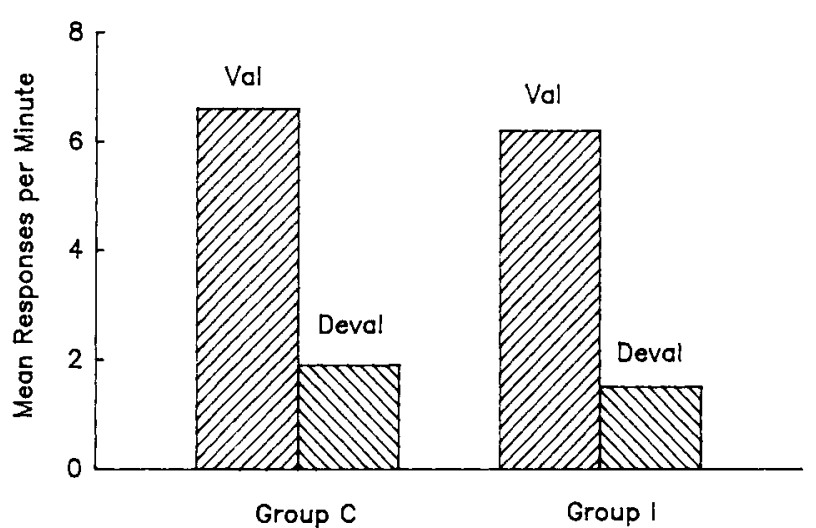

Figure 7. Mean responses per minute during the compound signal in the test session of Experiment 3. Performance is shown separately for the responses whose earned outcomes had been devalued and for those whose earned outcomes remained valuable.

the two test sessions with the two pairs of manipulanda. That figure shows responding in the presence of the discriminative stimuli, separated according to whether the outcome earned by the response had been devalued or not. It is clear that devaluation sharply depressed the likelihood of a response in both groups, and to about the same extent. The difference between responses whose outcome had been devalued or not was reliable in both Group C, $[T(8)=1, p<.02]$ and Group $\mathrm{I}[T(8)=0, p<.01]$. There were no reliable differences between groups. These results suggest that devaluation of an outcome earned by a response had a comparable disruptive effect in both Groups $\mathrm{C}$ and $\mathrm{I}$.

The pattern of responding during the ITI was similar to that observed during the compound stimulus, but substantially lower overall. The mean responses per minute were 1.0 and 1.5 for the"tesponses with devalued and valued outcomes in Group C; the corresponding numbers were .4 and 1.1 for Group I. The difference fell short of reliability for Group C $[T(8)=6, .05<p<.10]$, but proved reliable in Group I $[T(8)=0, p<.01$.

\section{Discussion}

There are two primary results of this experiment. First, a differential outcomes effect can be obtained under circumstances in which only the stimulus bears a differentially consistent relation to the outcome. It is not necessary to have differences between groups in the consistency of the $\mathrm{R}-\mathrm{O}$ relation in order to obtain a differential outcomes effect. Second, devaluation of an outcome after such a procedure has a substantial and equivalent detrimental effect on the response paired with that outcome, regardless of the consistency of the relation of the stimulus to the outcome.

These results seem more congenial to an account that emphasizes an $\mathrm{R}-\mathrm{O}$ than to one that emphasizes an $\mathrm{O}-\mathrm{R}$ association. The obtaining of a differential outcomes effect implies that the differential treatment of the stimuli 
was effective in controlling responding. Consequently, it verifies that the differential anticipations conditioned to the stimuli were operative during the discrimination training. In explaining that effect, the conventional two-process theory assumes that anticipated outcomes become associated with the response in the form of an $\mathrm{O}-\mathrm{R}$ association. But if it is differential $\mathrm{O}-\mathrm{R}$ associations that contribute to the superiority of the consistent group, one would expect a larger effect of outcome devaluation in that group. The failure to find that result encourages the possibility that despite the differential S-O associations, the associations between $\mathrm{R}$ and $\mathrm{O}$ are similar in the two groups. The natural way for that to occur is the formation of $\mathrm{R}-\mathrm{O}$ associations based on the consistent relation that each response bears to its consequent.

\section{GENERAL DISCUSSION}

The results of these experiments support the proposition that the formation of an $\mathrm{R}-\mathrm{O}$ association is a major way in which outcomes are encoded in instrumental learning. Experiments 1 and 2 arranged for a response to be followed by one $\mathrm{O}$ at a time when the Pavlovian arrangements ensured the greater anticipation of another $O$. They found, using a devaluation and transfer procedure, that it was the obtained, rather than the anticipated, $O$ that was better encoded in conjunction with R. Experiment 3 used the primary paradigm from which the presence of the alternative $O-R$ associations have been inferred, the differential outcomes effect. It demonstrated that in animals sharing the consistency of possible $\mathrm{R}-\mathrm{O}$ associations but varying in the consistency of the possible $O-R$ associations, there is an equivalent effect on responding of devaluing $O$. The differential anticipations were verified by the presence of a differential outcomes effect.

These results confirm and extend those of earlier experiments (e.g., those of Colwill \& Rescorla, 1985; Rescorla \& Colwill, 1989). Experiment 3 extends the devaluation effects previously reported by Colwill and Rescorla (1985). Their experiments were intended to match the anticipated outcomes during the training of two responses with different outcomes. This was accomplished by training both responses concurrently in the same environment. As a result, one would expect that both responses would be reinforced in the presence of the anticipation of both outcomes, thereby limiting the ability of an $\mathrm{O}-\mathrm{R}$ account to explain the devaluation effect. Nevertheless, differences in the local stimulus environment at the time that the responses earn their outcomes could be seen as supporting different outcome anticipations. For instance, the stimuli associated with the different manipulanda might signal the different outcomes in such a way that a particular outcome anticipation regularly precedes the reinforcement of a particular response. As a result, one could construct an O-R account of those findings.

A similar point can be made about Group I of the present Experiment 3. The arrangement of S-O contin- gencies in that group was intended to result in both responses being reinforced in the presence of anticipations of both outcomes. However, the combination of a signal and manipulandum might lead to the anticipation of one particular outcome, allowing only that outcome to become associated with the response. Nevertheless, the observation of less rapid discrimination learning in Group I than in Group $\mathrm{C}$ sharply constrains such an account. That difference in discrimination learning makes it clear that the groups must have differed in the outcome anticipations present during discrimination training. That is, the intention of giving consistent $\mathrm{S}-\mathrm{O}$ associations in Group $\mathrm{C}$ but inconsistent ones in Group I must have been at least partially successful. That the devaluation effect was nevertheless similar in Group I and Group C strengthens the conclusion that such anticipations do not account for the effects of devaluation on responding.

Experiments 1 and 2 are especially related to those reported by Rescorla and Colwill (1989), who attempted to pit against one another the $\mathrm{O}-\mathrm{R}$ and $\mathrm{R}-\mathrm{O}$ accounts by reinforcing a response with one outcome at the time that another was anticipated. The same strategy was used in the present Experiments 1 and 2, but with more substantial training in the presence of a Pavlovian signal of the alternative outcome. The higher rate of the unearned outcome throughout the course of discrimination training should ensure that the discriminative stimuli continue to be superior predictors of that outcome. Moreover, the development of stimulus control over the response documents that there was ample opportunity for the stimulus, as well as the anticipations that it controlled, to become associated with the response. The finding that it was the earned, rather than the unearned, outcome that governed the effects of devaluation and transfer therefore strengthens the prior conclusion that $\mathrm{R}-\mathrm{O}$ associations dominate $\mathrm{O}-\mathrm{R}$ associations.

It should be noted, however, that some of the results found in Experiments 1 and 2 could also indicate a contribution of $\mathrm{O}-\mathrm{R}$ associations to performance. For instance, in Experiment 1, the devaluation of the earned outcome, which left intact the value of the Pavlovian outcome, failed to remove fully the responding during the stimulus. Moreover, in Experiment 2, a discriminative stimulus that signaled the outcome delivered on a Pavlovian schedule also elevated responding, although less than that signaling the earned outcome. One interpretation of these observations is that an $\mathrm{O}-\mathrm{R}$ association is also formed and can contribute to performance. However, it is also possible that these effects instead arise from an unintended $\mathrm{R}-\mathrm{O}$ association. It should be noted that although the Pavlovian outcome was delivered during the stimulus independently of responding, there is an important sense in which there is a contingency between the response and that outcome. The Pavlovian outcome only occurred during the discriminative stimulus; moreover, because of the response contingency arranged for the other outcome, the response was also substantially more likely during the stimulus than during the ITI. It therefore fol- 
lows that the likelihood of the Pavlovian outcome is higher at the time of the instrumental response than in its absence. Although the procedure did not deliberately arrange it to be so, it is clear that the contingencies provided the opportunity for the formation of an $\mathrm{R}-\mathrm{O}$ association between the response and the Pavlovian outcome. It is possible that this is the basis for the continued level of performance in Experiment 1 and the moderate transfer effect based on that outcome observed in Experiment 2.

Finally, the present results have implications not only for the interpretation of conventional instrumental learning experiments but also for the more complex procedures normally used to demonstrate a differential outcomes effect. They show that the effect does not depend on different $\mathrm{R}-\mathrm{O}$ associations, but can occur when only the stimulus is treated differentially with regard to the outcomes. For that reason, they encourage the view that a differential outcomes effect may result because of variations in the degree to which an S-O and an R-O association share the same $O$.

But the principal conclusion to be drawn from these experiments is that the $\mathrm{R}-\mathrm{O}$ association is a primary means whereby the outcome is encoded in instrumental learning.

\section{REFERENCES}

Colwill, R. M., \& Rescorla, R. A. (1985). Post-conditioning devaluation of a reinforcer affects instrumental responding. Joumal of $E x$ perimental Psychology: Animal Behavior Processes, 11, 120-132.

Colwill, R. M., \& Rescorla, R. A. (1988). Associations between the discriminative stimulus and the reinforcer in instrumental learning. Journal of Experimental Psychology: Animal Behavior Processes, 14, 155-164.

Peterson, G. B., Linwick, D., \& Overmier, J. B. (1987). On the comparative efficacy of memories and expectancies as cues for choice behavior in pigeons. Learning \& Motivation, 18, 1-20.

Peterson, G. B., Wheeler, R. L., \& Armstrong, G. D. (1978). Expectancies as mediators in the differential-reward conditional discrimination performance of pigeons. Animal Leaming \& Behavior, 6, 279-285

REsCoRLA, R. A. (1990). Instrumental responses become associated with reinforcers that differ in one feature. Animal Learning \& Behavior, 18, 206-211.

Rescorla, R. A., \& Colwill, R. M. (1989). Associations with anticipated and obtained outcomes in instrumental learning. Animal Leaming \& Behavior, 17, 291-303.

TRAPOLD, M. A. (1970). Are expectancies based upon different positive reinforcing events discriminably different? Learning \& Motivation, 1, 129-140.

Trapold, M. A., Overmier, J. B. (1972). The second leaming process in instrumental learning. In. A. A. Black \& W. F. Prokasy (Eds.), Classical conditioning II: Current research and theory (pp. 427-452). New York: Appleton-Century-Crofts. 